L'importance d'un tel fait, qui semble corroboré par les espériences que nous venons de décrire, est de premier ordre pour l'emploi de l'ajutage Venturi dans le cas des essais des turbines hydrauliques, de débits un peu considérables, et en général de tous appareils hydrauliques très puissants.

L'intérêt qui s'attache à cette question ne peut échapper i personne, et elle mériterait certainement une étude spéciale sur d'aumes types d'appareils. Nous espérons vivement que loccasion nous sera fournie de revenir sur ce sujet plein d'intérêt; nous nous permettons d'attirer sur ce fait l'atten. tion de la Commission des Turbines; à la générosité de laquelle, comme nous l'avons déjà dit, nous devons ces preniers résuluats.

Essais divers. - Essai des pelits compteurs d'eau. A l'extrémité de la salle des essais hydrauliques, près de la rampe d'accès pour l'arrivée des machines, nous avons installé deux groupes d'appareils pour la vérification et pour les essais des compteurs d'eau.

Le premier de ces appareils, fourni par la Compagnie pont la fabrication des Compteurs, se compose de deux réservoirs à niveau constant, alimentés par l'eau de la ville, quidonnent respectivement des charges de 1 et 2 mètres.
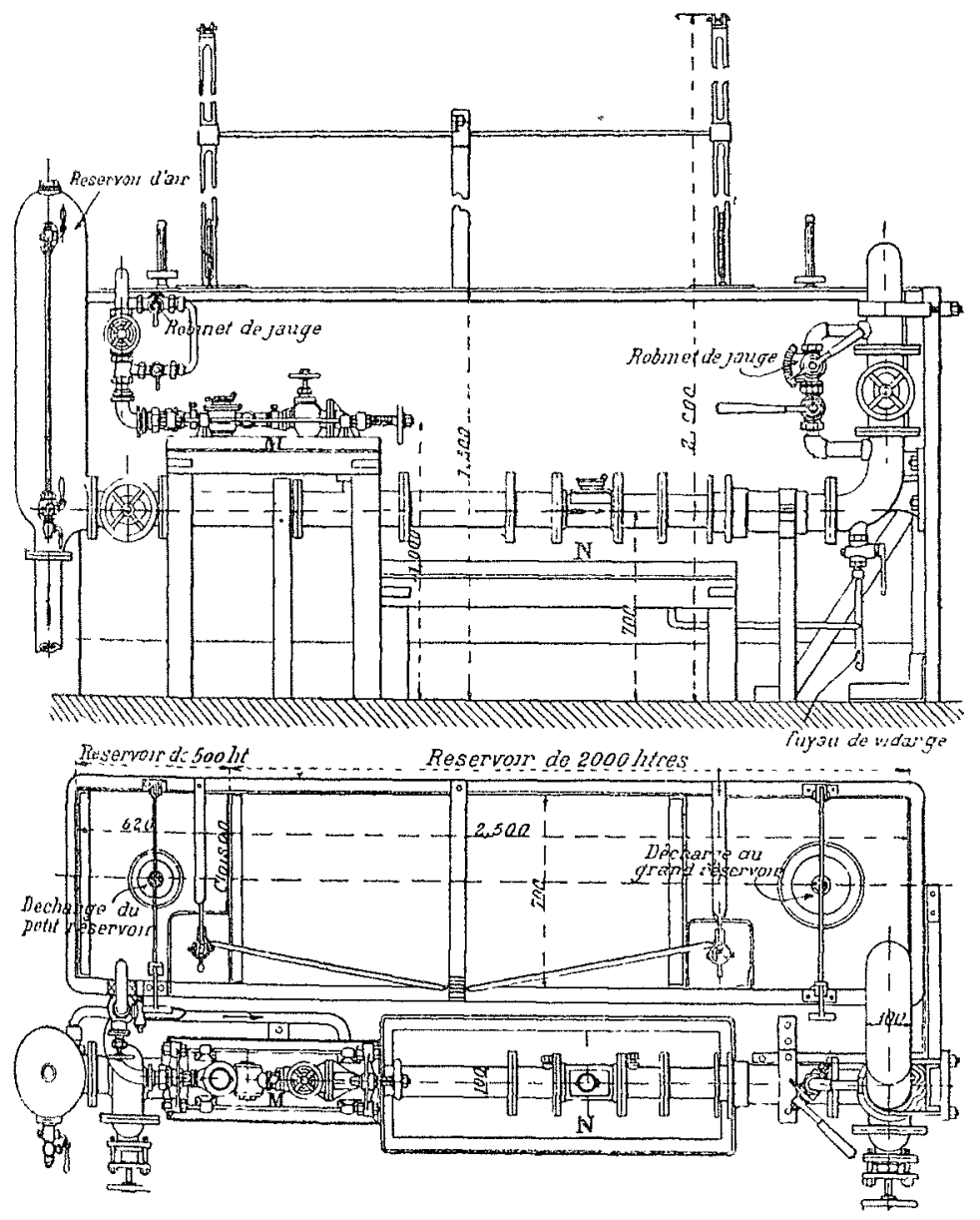

Fir. 26. - Appareil pour l'essa des compleurs d'eau.

Par un jeu de vannes et de robinets, on met en communication l'un de ces deux réservoirs avec les compteurs à essayer. L'eau évacuée par ces derniers est mesurée dans des bâches jaugées de i io litres. Les débits sont réglés par des diaphragmes calibrés, et la jonction des compteurs à l'appareil d'essais est réalisée par des tubes souples en caoutchouc.

\footnotetext{
i Monimatre line serie de mesules qui lu ont permis de constater que le debit de cet instrument calcule theorlquement élait tres rapproche du debit vra. Revue de mécanique, septembie 1907 .
}

Ce dernier appareıl peut également être mis en communication directe avec l'eau de la ville, et l'on opère, au besoin, avec la pression totale de cette dernière.

Les compteurs sont aussi essayés, surtout pour les débits un peu plus importants, avec un appareil construit par la Compagnie générale des Conduites d'eau. Il est disposé en vue de mesurer non seulement le débit, mais encore la perte de charge.

Le compteur est mis en place sur un banc d'essai (fig. 26) au moyen d'un dispositif qui rend la manceuvre rapide et commode; l'eau quile traverse est recueillie dans des bàches à flotteurs; une échelle graduée indique à chaque instant la consommation en eau. Un manomètre hydraulique différentiel $\mathrm{P}$ permet la lecture des pertes de charge pour les différents débits. Les compteurs au-dessous de $40 \mathrm{mili}$ metres sont essayés en $M$; ceux de 50 à 100 millimètres, cn $\mathrm{N}$.

Appareils pneumatiques. - Une canalisation d'air comprimé à $12 \mathrm{kgs}$, qui court le long d'un mur de salle, permet de faire l'étude des moteurs à air comprimé, des freins, outils pneumatiques ou autres appareils.

Appareil d'essai des compteurs à gaz. - Enfin, dans cette salle, et dans un local complètement séparé, est installé un appareil pour l'essai des compteurs à gaz, il se compose d'un gazometre de 500 litres, entièrement équilibré, quion peut remplir d'air ou de gaz. Ce gazomètre est en communication avec les compteurs à essayer, qui sont placés parfaitement d'aplomb sur un marbre. Le gaz entre et sort du compteur par des conduites en caoutchouc munies de joints hydrauliques. Ce banc permet l'essai de six compteurs à la fois. Des manomètres mesurent la perte de charge dans la traversée du compteur. Après avoir circulé dans tous les compteurs montés en série, le gaz passe dans un compteur étalon qui permet de contrôler les lectures faites au gazomètre; il se rend enfin à une rampe, où il est brûlé dans une série de becs papillons installés sous la hotte.

Boyer-Guillon, Ingénieur civil des Mines,

Chef de la section des essais de machnes au Laboratore ¿'Essats du Conservatoure National des Atts et Métier's.

\section{LES CANALISATIONS SOUTERRAINES}

Communication fate au Congrès de Marseille par M. Dr MARCHriva, Ingénieur en chef do la Compagnie Thomson-Houston.

Les canalisalums souterranes, le syslimes si diver's aux debuts des apploatıons électriques, dérivent maintenant tontes d'un lypo unique, celuu criog il y a quelques vingt ans par Siemens. Tos perfectionnements de détals appoltés it ce type lonl amenó it un point do perfection qui a permis d'ctendre singuhirement lo champ de ses apphoations, et, a l'heure achelle, ces dernledes compoltenl des voltages ruu, il y a peu de temps, étaient considirés comme représentanls les limites extrumes admissibles pour los inslalla iinns électriques.

Limportance tris consiclérable qu'a prise cette fabricalion sprochale rend parficullerement disirable de fixer a son sujel quelques liggles proises, qu puissent servit de bases of do guides ansst ben anx acheleurs qu'aux foumusseurs. Le sujeb est maintenant suffisamment commu, ol les dombes l'expríriences assez nontbreuses el caractéristiques, pour que ces règles puissent otre traries sans trop l'meerlifudr.

\section{I. -- CONSTITUTION DES CANALISATIONS SOUTERRAINES}

On peut comsiderer les rables soulerrains commo ronshlues de trois parlics netloment dislmctes : a) Les conduclenes de fraval ; - b) L'enveloppe profeclice exterieure ; a) Tikntant sóparant les comductours de l'onveluppe protectrice. 
conducleurs. - Tes condicteurs de haval peuvent the mintles ou multiples (2 ou 3 sous la mène enveloppe). Dans ce denuer cas, lls sont presque tonjours à theme actuelle, conshlués par des; cables en curve synéhiquement placés et enroulés en héhces it pas plus ou mons allonge. On a a peu prés renoncé a linsage des cables concentriques, meme dans lo cas des chbles in dewx conclucteurs. Ces citules sont composés de lorons it sectirn sembiblemenl

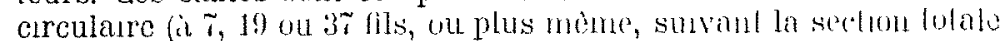
a réaliser); et quoldue, pour meux uhliser l emplacement dispo. nible et róduire le dramcitre nutineur, on at parfors remplacé Jes torons à section circulare par des fansceaux à section scusiblement triangulaire, on peut dire que celte maniere de farre ne s'est pas rupandue par sule de ses jnconvintents praluques, henuconp plus grands que l'avantage cherche.

D’une mantire génirale, les conduclens on cuvie sonl ilamis

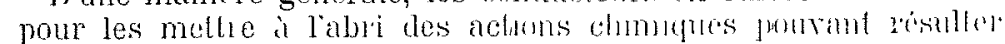
din contact avec les matieres isolantes.

Enveloppe prolectrce. - L envelopme prutectuee exténeure jour

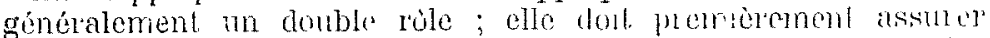

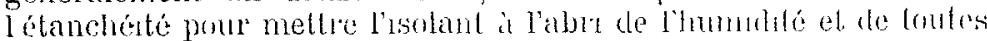

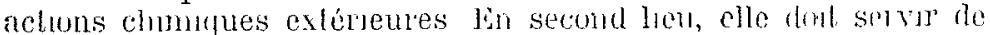
protection miranufue, non seulement apres lit pose, mas encom durant les upírations de tramspot t of de mise an place.

Litanchértít est obtenue it larke d une on de deur games de plomb contmues, d'epansseurs plus ou mons graules ; la protection mécanlque est réaliséc sont par the enveloppe de lils d'acier, sont plus genétalement par une double enveloppe an lubans l'acier: Un matelas de lilm soudromné est merposé ontre les ganes de plumb of les rubars dacter ; gidninglonent in autre matelas somblable forme l'enveloppe catérieure du cible.

la vie du cable chand déterminée par la resistance de l'enveloppe en plomb aussi bien nux agents chmmunes yu "a l'dectrolyse et and

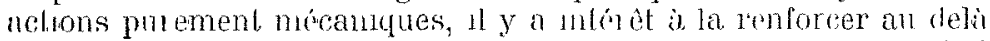
le co qui seral necessare pour assurer stretement l'ilanchíle inuliale. I l'heure actuelle, on ne descend pas au-ressous de? $\mathrm{mm}$. l'óprisseur pour l'envelompe en plomb. et on lui dome générnlemont entre :i et í mul. Ansist (et pruncipalement pour los cillies

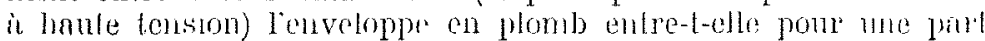
très importante dans te prix do revleul folal des cibles anmés.

pour certames applications spectales, le plomb a dé lemplace

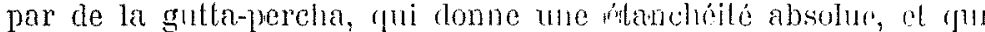
sert a la fois d'isolant et d'enveloppe prolectrice. Mars ces apyli-

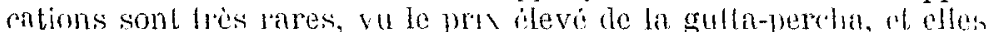

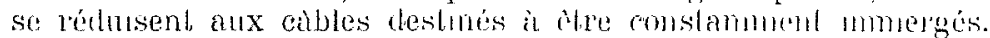

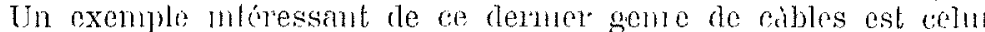

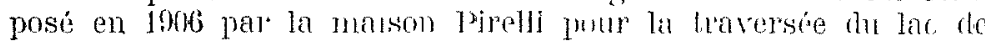

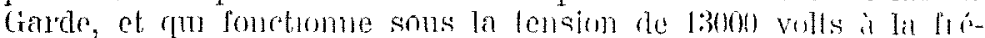
quance de to pórionles.

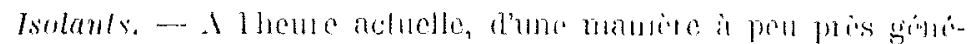

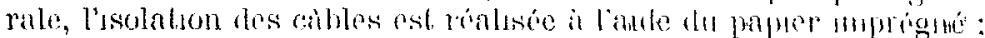

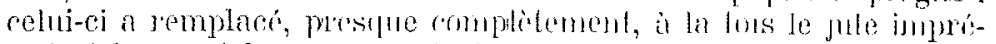

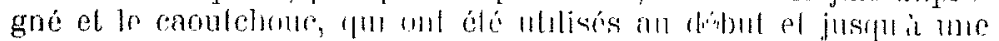
rlizaine d'emnérs.

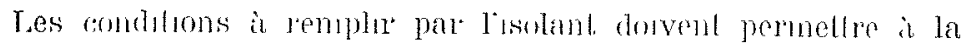
fors d'assumer un bon fondionnement of do tialiser un prix de rovient moderé. Ces conditons sont mulluples. Il dul tout diabord posséder une risistance flílectrique considirable ; - n dul cho parfatement homogine el rigglier ; - il doil dre stable, cost-itdire eouserver toules ses qualités, quel que solt la fluróo do som usage, ol dans les condilions physigurs varres ; - 11 houl pos-

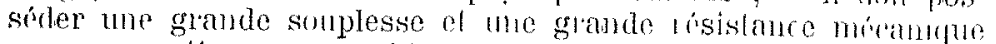

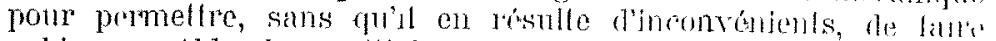
subir aux cibles les multiples manulenloms qu'exigest lent labrt-

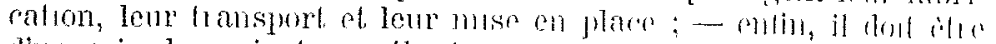
d'un prix de revient pon allevi

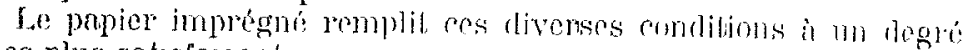
des plus satisfarsants.

Dans ces dernières ammes, des éludes approfondies ont eté fates qui ont donné lieu à des améhorations considérables, grice an choix judicuenx des matières employées d de lentrs dosages. Yaturellement, chaque fabrimant conservo secrels, d'wne manice

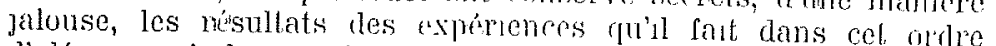
d'udes ; mais la grande simihtude des prodults obtenus dans les rliverses masons de premier ordre montre que les rigles de la fobrication ne doivent que bien peu s'écurte de l'me i lautre. La condition essentielle du surers ristele, dans fous les cas. dans mon

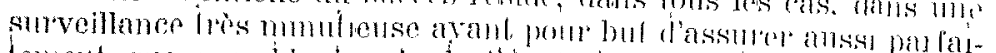

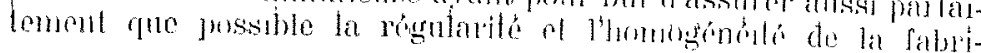

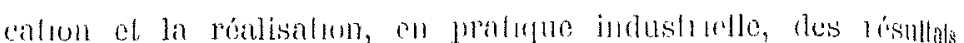
oblems au laburatome.

Limprégnation des cables se taul apres que cenx-cr ont fít soj. gineusement séchés par l'action combmée de la chaleur ol du vile,

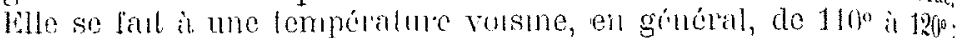

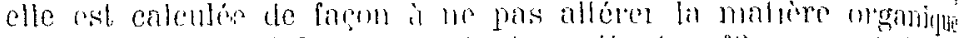

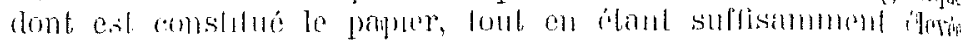

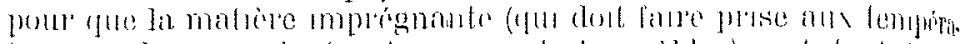
tures ordmances de fonctionmemont des cables) sult foul it lant flucle lors de l'mprégnation.

Dans ces denlucres ammés, en vue de la fabrucalion des cibleş a trìs haul voltage, on a propusé de divers eólís une consfotullint?

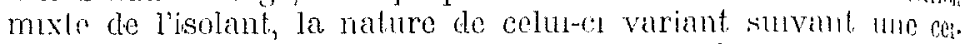

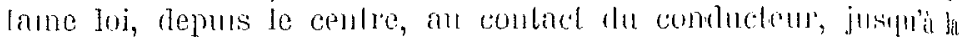

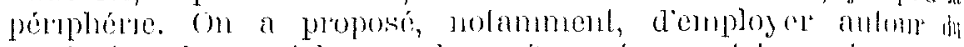

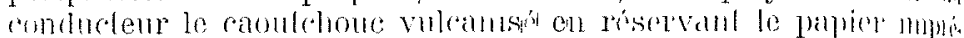

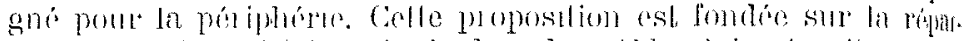
fithon du polentred tres migale dans les cibles it haul vollage cron

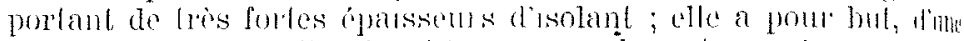

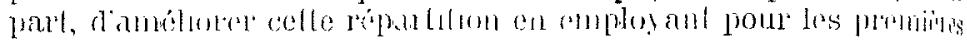

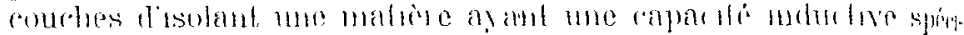

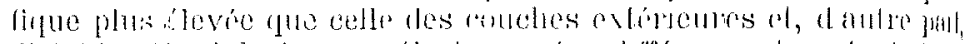

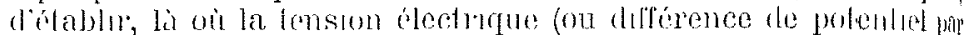

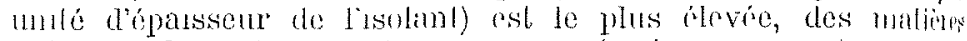

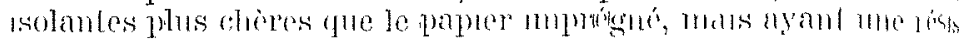
tance dielechrugue plus grande

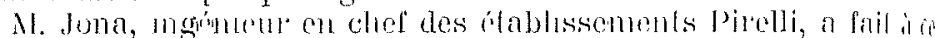

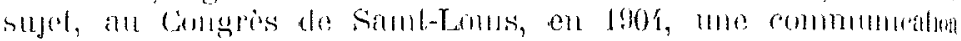

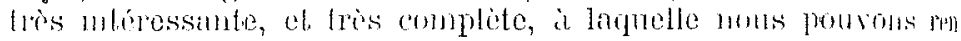

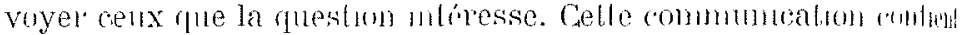

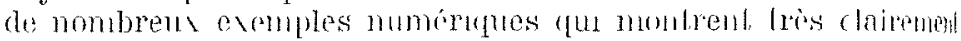

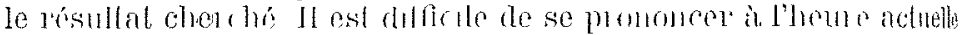

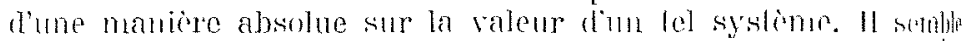
que dans la giande mapolde des cas les avanlages pratumes eil soreut bren mommes of compensés par bien des monvinumb.

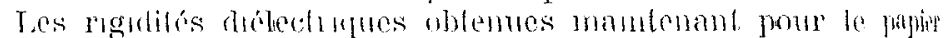

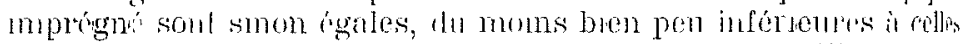

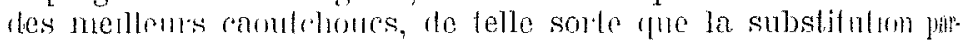

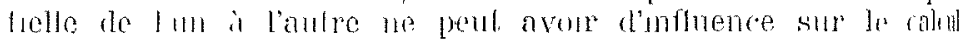

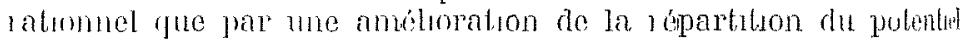
ben peu somsible aux basses of moyemnes tonsions. T'aulre jat,

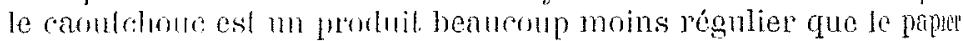

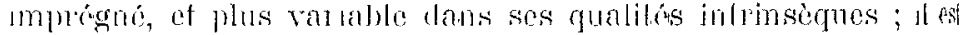

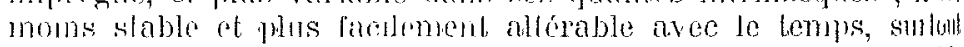

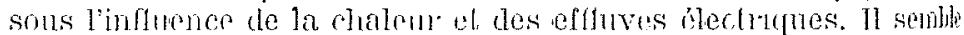

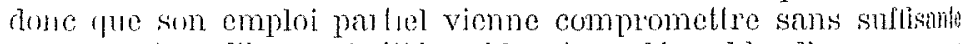
compensalom l'homogingétí ru'il est si désmable d'assurer allt cibles sontertains. Pour le moins, on poul dire qu'en ce qui con corne les vullages infirieurs it 12004 on 13000 volls 11 conshlue un

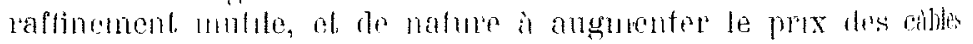
plubl qu'it le rémine

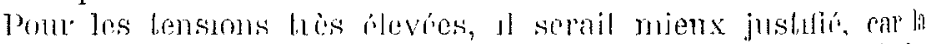

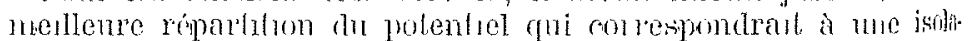

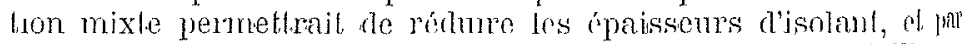
sute le prix de l'enveloppe en phomb el de l'armabure mélalliglue: l'économie flui en résulted all poumal, dans cedtains cas compensel el au delì la plus-value résultank de l'emploi parliel du caut-

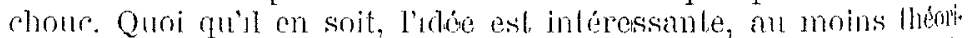

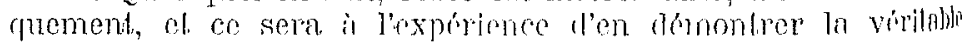
valeur pratique.

$\Lambda$ vant de termmet la ruestion des isolunls, nous devons signalen 111 procédí qui a pris ces dernuers lemus une nsse\% grande extent stron en Amiriquo : nous vonlons parter des cubles it isolanls dits larnivhed Cambric Ces cables, dont la fabricalion dale te 19in?

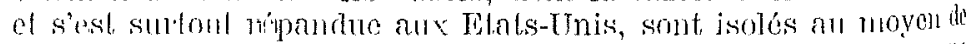

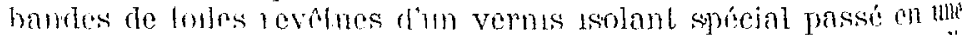

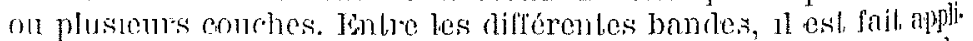
calion diune munce enche d'une matiere ssolante plastique, ignle. ment de composition spéciale, of qui assure l'arthérence des difle rentes bandes veruissees entre elles, toul en leur permellant all besoin de glisser un pen l'une par rapport i l'autre Cet onduit spreial a en onlre pour effel d'empechel l'absontion par eapillarilt de l'humidite ot la prósence de pelits intervalles d'air entro les couches. Charquo bamde rle trile vernissé, d'me epaissemr de un

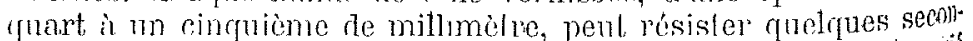
des a mo lension de 8000 it 10000 volls, el Ieur nombre est vitip sulvaml le vollage anquel le cable dot travailler.

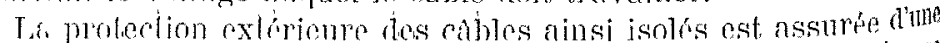
maniere difforente suivant leur destination (tresse ondmare, tresse 
damante, enveloppe en plomb avec ou sans armature de feulfard) ; los diverses míthodes applicables aux cảbles au papier et aux cables au caoutchouc sont également employées pour ces aubles spéciaux. Ces cables sont d'un prix de revienl plus élevé que les cibles au paprer, mass lls présentent pour quelques apphcations, et notamment pour Ies connexions intérieures d'usmes, cartans avanlages qui, d'après les fabricants, sont surtout les sulvants: $1^{\circ}$ Plus grande régularité de la fabrıcation et plus d'homogénich lé pour la résıstance à la tension résultant du mode même de fabrication ; - 20 plus de llexiblilté des matières composant jisolint. Cotte flexibililó permet de cmtrer les cables cambric sins un rayon rédut à six fors le dianètre exténeur du càble. Llle est pricueuse pour les canalisations intérteures ; - $3^{\circ}$ Plus grande réssistance à la chaleur que le caoutchouc, et à l'humıdité que les ribles atu papier implégné.

Les cables cambric se fonl. aussi du système composite pour les tris hantes tensions, c'est-à-dıre avec isolation au caoutchouc au volsinage du conducleur el à la tolle vermssée dans la partie extéleure. Ces calbles Varnished Cambrc donnent lıeu à des phénomines d'lyystérésis diélectrique plus marqués que les câbles au papter et sont, de ce chef, plus exposés à s'échautfer lors des twsars sous très haute tension.

\section{MISE EN PLACE DES CANALISATIONS SOUTERRAINES}

Deux systimes sont en présence pour l'ćlablissement des canalisallons soulerrames.

Le prenner systeme, dit des cables armés, est employé d'une moniere à peu près exclusive en France, en Italie et en Allemagne ; 1 consiste à poser les câbles munıs d'une armature métalligue exterieure a meme dans les tranchées ouvertes dans la dirussce. Génćralement, les cables sont posćs sur un lit de sable, on the scule nappe, $\dot{a}$ une profondeur d'au moms $0 \mathrm{~m}$. $i 5$, et, en outre de leur armature prop.re, lls sont protígés, solt par des grillages ctablis au-dessus de la nappe, et qui en signalent la présence lors des travaux de foulle les meftant à nu, soul par un cours de planches ou de briques cassées, solt par les deux moyens rónis.

Co syslome a l'avantage dêtre d'une exécution tris simple et trìs lacile; ll se prête facllement à l'établissement des boites de jonchion et de dórivation et, de ce chef, est adoptét à l'exclusion de tout autre pour les cables de distribution. Il permet d'emplover de plus grandes longueurs de cables d'un scul tenant, et évite d'avorr à calculer à l'avance avec prícision les valeurs qu'll convient de domner à ces Iongueurs.

D'autre part, l'armature mćtallique est très précieuse pour proléger le cable durant les opérations d'enroulage, de déroulage et de tirage. Grace ì sa raideur, elle soulage de tont effort l'enveloppe en plomb et les couches isolantes durant les divers cinl'ages auxquels donent lieu les diverses mampulations du cảble. Enfin, elle constilue une protection sérıeuse de l'enveloppe on plomb contre les actions chimiques et l'électrolyse.

Le second systime, dut solnd system ou conduit system, est sur tout employé ien Amérique, en Angleterre et ses colonies, et, d'urie manière gónérale, dans les pays anglo-saxons.

Il consiste da tirer les câbles (simplement revetus de leurs gaines de plomb) dans des conduits cylmdriques de types varıés. De distance en distance, des regards et des fosses de visite sont ménagés sur le tracé des canalisations pour permettre le tirage des cables et l'ćtablissement des jonctions. Celles-ci se font généralemenl au moyen de manchons en plomb, de plus grand diamètre que les cables, et soudés à Jeurs gaines extérieures. Les fosses sont munies de tampons d'accès et diévacuation à l'ćgout. Quant aux conduits, lls sont généralement enrobés dans une maçonnerıe de béton qui en font un bloc unique, percé d'alvéoles correspordant a charue cable.

Les avis sont tròs partagés sur les avantages et les inconvénients de ce système. Son pruncipal avantage est de réduire l'en combrement en plan occupé par les canalisations. Il permet, "n onlre, de néserver la possibilité d'ajouter de nouveaux câbles sans avoir de nonveau à ouvrir la chaussée ; il suffit de réserver les alvéoles correspondantes lors de l'exécution des conduit is. De même, les réparations et les remplacements peuvent s'elfectu: sans ouverture de tranchées. Les remplacements d'un cable avarić ou défeclueux peuvent donc s'effectuer avec de très falble frais de pose, et l'on pcut récupérer le prix de vieux matériaux entrant dans ce cable (plomb et cuivre) sans avoir a payer des frais élevés de reprise, c'est-â-dire avec le minimum de déchets. Cette facilité permet d'admettre un taux d'amortissement moindre pour les cables posés de cetle façon.
L'absence de loute armature sur l'enveloppe en plomb permet, au moment du trage, de constater très lacllement et avec une grande certitude, le bon état du cable, les momdres défauts et meurtrissures s'apercevant tris bien sur l'enveloppe en plomb quand elle reste ì nu. Ce systeme olfre entin unc protection presque absolue contre les accidents mécaniques postérieurs à la pose.

Les mconvénient sont : Le coùt sensiblement plus élevé de ces canalisations, surtout quand il y a peu de cables (moms de 1 à 6 ) à tirer dans la mòme iranchée ; - les précautions plus grandes a prendre pour le transport et les manutentions diverses de cables non anmés; - les déchets plus grands au moment de la pose, par surte des longueurs strictement fixées que dolvent avon les dilférents tronçons entre deux jonctions ; - les chlficultes de trages auxquelles donnent heu les changements de direclion entre les deux fosses de risite ; - la inoms facile radiation de la chaleur, et par sule léchauffement plus grand des cables.

Ce système parait plutòt indiqué pour les canalisatıons imporlantes à établır dans les villes à rues cétroltes, régulières, consLituant des ilots de dimensions similares telles qu'on les trouve clans les cilés nouvelles des pays anglo-saxons. 11 constutue meme la seule solution possible quand le nombre de cables à poser lans la même rue devient très considérable.

- Les conduites employées sont de divers types : grès, poleries ou fibres spécıales. Ces demières sont plus coùteuses, mas scmblent présenter, par leur légèretí, la longueur la plus grande de leurs tronçons et la meilleure prolection qu'clles ollrent contre l'óloctrolyse et les courts-circuits, des avantages qui tendent à en nipandre l'emplox.

Il est assez difficile de chlfrer d'une manière précise la différence de dépenses ì envisager dans l'un ou l'autre systime, car cotte dilférence dépend beaucoup des condrlions partıculic̀'es à chague installation. Toutefors, on peut dire qu'clle varie preu avec Ie nombre de cables constıluant la canalısation, et qu'en morenne elle peul osciller entre 3000 et 6000 frs par kilomètre de canalisation. Celte différence très appréciable quand il n'y a qu'un ou deux câbles à poser dans une môme tranchée, devicnt beaucoup moms mportante quand ce nombre s'olève it 6 ou it 8 .

Un autre bon système i employer dans le cas des cables armés consiste à les dérouler dans de petıls conduits en boıs remplis cnsuite d'asphalle. On oblient ainss une excellente protection contre l'électrolyse et les achons chimiques, et anissi un supplément appréciable de séducilé contre les accidents mecamaues. Cette disposition, tròs recommandable dans tous les cas ou l'électrolyse est à craindre, peut entrainer un supplíment de dopenses d'environ 800 ì 1000 frs par kilomètre de cable.

Quel que solt le systeme de canalisation adopté, les câbles souterrams principalement ceux au papler, demandent à elre posés avec corlaines prícaulıons. J'une mantère génćrale, colle pose ne doit etre confiée qu’à des ouvriers spécialıstes bien habulués à co genre de travali ; les cables dorvent etre brutalisós le moins possible, et il faut éviter de les cintrer en aucun point sur des rayons trop courts. Les boites de jonction dorvent otre suffisamment vasties en proportion des voltages adoptés et etre remplies avec soin, en tenant compte du retrait que prend la maticre isolante en se refroidissant.

Le papier mprégné devient rapidement plus dur et plus cassant au fur et à mesure que la température s'abaisse. Ausai est-l très important de ne pas faire la pose cle câbles au papier a des tem"sratures basses : $6^{\circ}$ a $7^{\circ} \mathrm{C}$., doivent etre considérés comme un minimum, et, surtout pour les cables a haute tonsion, il est préérable de rester plutot au-rlessus de $10^{\circ} \mathrm{C}$. Quand les circonstances obligeront absolument a farre la pose de cables 4 des tompératures inférieures it $6^{\circ} \mathrm{C}$., il faudra avorr soin, avant d'uthliser les bobines, de les conserver dans un endroit convenabloment chauffé assez longtemps pour que la température se solt élevéc dans toute leur masse au niveau de celle de ce local. Les conditions climatériques les plus favorables se rencontrent vers $25^{\circ} \mathrm{C}$. ; les températures supérieures sont également avantageuses, mais il faut prendre garde, a ces temperatures, que les differentes spires des bobines n'adherent trop les unes aux antres ct observor, de ce chef, quelques précautions au déroulage.

Mise à la terre du point neutre. - Avant de terminer ce chapitre nous dirons quelques mots sur la question très controversée s'il convient ou non de mettre a la terre le point neutre des réseaux de cables souterrains.

La plupart des fabricants sont favorables à l'adoption de cette mesure ; mais quelques-uns, et non des moindres, y sont forté- 
ment opposés. Si nous exammons la question au seul point de vue des cables, nous constaterons que la mise a la terre du point neutre donne plus de stabllité aux conditions électriques de fonctionnement ; elle diminue beaucoup les di!térences de potentrel possibles enture conducteurs et terre, ce quı permet de réduire en proportion les épaisseurs d'isolant entre conducteurs et l'enveloppe en plomb, c'est-à-dire en définitive le coût du câble.

Toutefois, pour que cet avantage solt plemement réalisć, il faut que la mise à la terre solt franche, c'est-di-dire que la résistance de cette connexion solt assez fable pour empecher que jamais er aucune crrconstance la dittérence de potenliel entre point neutre et terre ne puisse prendre une grande valeur. Il en résulte de cet inconvénient que, en cas de mise à la terre d'une phase par claquage de lisolant (par exemple à la suite dun accident mécanique ayant affecté lisolant en un point), un courant intense peut passer par le défaut et la connexion à la terre en crrculant à travers l'enveloppe en plomb, et y causer souvent de graves dégàts, lesquels se révéleront ultérieurement par d'autres claquages.

Le danger est surtout grand avec les cables à basses et moyennes tensions, pour lesquels les sections des conducteurs et les intensités des courants qui peuvent y circuler sont mportantes par rapport aux dimensions de lenveloppe en plomb, et pour lesquels aussi les appareils de sécurité (fuslbles, déclencheurs, etc.) sont réglés pour des intensités considérables, et sont parfols susceptibles de ne pas fonctionmer en cas daccident sur une phase. Comme, r'autre part. aux bisses . nt noyemes tensions. les réductions sur lépasseur des conches isolantes rendues possibles par la mise à la terre du point neutre sont très mmimes et d'une influence presque nulle sur le coût du cable, cette mise à la terre n'offre guère d'intérêt.

Pour ces raisons, nous croyons préférable de fonctionner avec le point neutre isolé toutes les fors que la tension de service sera inférieure à 6000 volts. La situation change au fur et à mesure que la tension de service s'élève, et, il partir de 10000 ì 12000 volts. nous considérons la mise à la terre du point neutre comme tout a falt désurable et sans inconvément sérveux. Il est mtéressant de remarquer que cette mise à la terre conduit ì supprimer ou tout au moins à rédure considérablement la couche disolant qu enveloppe l'ensemble des trois conducteurs. Cette suppression est très avantageuse, car c'est précisément cette couche d'isolant qui se trouve le plus fatiguée lors des manutentions du cable et de ses cintrages sous de falbles rayons ; cest par sute celle dont l'efficacité a le plus de tendance à dimmuer pendant les opérations de mise en place.

Les differents conducteurs dun cable a conducteurs multuples vitant enroulés en hélice, leurs enveloppes isolantes individuelles ne tendent au contrare pas a sallonger ou se raccourcr les unes par rapport aux autres du falt d'un cintrage de l'ensemble, et elles sont par suite soumises a des fatigues beaucoup moindres. Ce fart ost suffisament important pour qu'll nous paraisse préférable, meme quand leur pont neutre n'est pas mis à la terre, de supprimer toute enveloppe isolante extérieure sur ies câbles devant fonctionner sous tensions égales ou mférieurs à 6000 volts. Audessus de 10000 a 12000 volts, la possibilité de cette suppression đevra ètre consıdérée comme un avantage spécial à revendiquer en faveur de la mise ì la terre du point neutre.

\section{III. - ESSAIS DES CABLES SoUTERRAINS}

Ces essais dorvent servir, dune part, au controle normal de la fabrication et, d'autre part, a tixer les conditions de néception, cest-i-dire a concller les points de vue différents du fabricant et de l'acheteur.

Le fabricant a pour désır naturel de limiter sa responsabilnté. et de se borner à vendre, pour un prix déterminé, un objet bien déterminé. L'acheteur a surtout en vue l'usage qu'll compte faire de l'objet acheté et désire que cet usage lu donne entière satısfaction. La concurrence commerciale, en poussant à l'extrême la recherche de la réduction des prix, a pour conséquence naturelle de tendre à empecher ces deux objectifs de se confondre, et il en est résulté trop souvent, entre les fabricants et leurs clients, de graves contestations où cependant la bome foi se trouvait des deux còtés.

En principe, l'acheteur, quand il y a mis le prix, est en droit de demander que le matériel acquis par lui fonctionne en toutes circonstances d'une manière normale et sans accidents ; c'est au fabricant, mieux au courant des limites qui conviennent à lusage de ses produits, qu'll appartient de fare le nécessaire pour qu'il en soit ainsi. Il ne doit jamais perdre de vue qu'en aucun cas la sécurité du fonctionnement ne doit être sacrıriée à une question de réduction du prix de vente. Ce principe ne peut cependant etre poussé à l'extreme, car les accidents peuvent aussi provenir de taits anormaux ou de fautes graves commises par l'acheteun, dont le fabricant ne peut toujours porter la responsabilité.

bans l'mdustrie des cables souterrams, la question est d'autant plus délicate qu'il est plus difficile de tracer une ligne de démar. cation précise entre ce quı devra être considérié comme un fait normal et ce qui pourra, au contraire, etre considéré comme vraiment anomal et exceptionnel. D'autre part, la recherche des responsabililés à la sulte d'un accident est toujours très difficle, l'accident ayant généralement pour premier eflet de faire dispa. raitre toute trace de son origme premièrè et entrainant avec lui des conséquences qui peuvent, à tort, être confondues avec les causes.

Il cst donc logique de soumettre les cables souterrams a des essais ayant pour objet d'indıquer nettement à l'acheteur le degrí de sécurité que leur emploı peut présenter et de limiter par sujte, dans une certame mesure, la responsabilité du fabricant.

Les essais de vérıfication ordmarrement prescrits portent : sur la résıstance kilométrique d'ısolements et sur la résıstance à la rupture, ou rigidıé dıélectrıque de lisolant.

Essais disolement. - L'utılité de ces essais est très contestéé. Avant de la, discuter, il convient de se rendre bien compte de la nature des renselgnements qu'lls peuvent fournır.

Ces essais ont nour objet de déterminer quelle est l'mportance du conrant qui, après une durée d'électrification détermınée, sous une différence de potentiel constante et bien délerminée, passe dans lisolant d un cảble. On peut remarquer de suite que cotte mesure n'a guère dintérêt au point de vue de l'usage mème du cable. En elfet, avec les courants continus à voltage modéré, lit perte à vide correspondant aux isolements ordinarres des cables est tellement réduite, qu'on peut la considérer comme absolument mappréciable par rapport au courant traversant les conducleurs.

Il en est de même, quolque à un degré moms marqué, avec les cables a haute tension ; mais, en outre, ces hautes tensions sont généralement alternatıves, et les phénomènes qui se passent alors n'ont quiun rapport élorgné avec ceux auxquels donne heu le courant contmu. Cela tient à ce qu'un régime perman:nt ne s'établit pas aussitot après qu'un cable est soumis à une diffir rence de potentiel déterminée, ni meme après la période d'elertrıficatıon de 1 à 2 minutes quı précède généralement la mesure de lisolement. Celle-ci se fait dans une période de régme variable, et l'allure de cette variation peut changer sensiblement dun cas à l'autre. Les données que procurent les mesures d'isolement sont donc d'ordre tıès complexe et n'ont aucune signification physigue bien précise

D'autre part, la raleur de l'isolement varie dans des limites très étendues avec la tompérature, et, quoique des Tables de correction solent employées pour ramener les mesures faites a une mème température, la correction ansi faite n'a guère de pré. cision, parce que les Tables de correction sont loin d'etre exactes pour tous les cas, et aussi parce que la température véritable à appliquer est souvent difficıle à connaitre avec précision et laisse planer beaucoup d'incertitude sur l'inexactitude de la correction. Les résultats fournis par les essais d'isolement ne doivent done être interprétés qu'avec une certaine méserve. En réalité, ces essais dolvent être consıdérés surtout comme pouvant servir de controle empirique de la fabrication, et iencore devons-nous ajouter que ce controle ne peut porter efficacement que sur la nature des matières isolantes employées, et non pas sur leur plus ou moms bonne mise en ouvre, les défauts de fabrication inte. rieurs pouvant fort bien passer maperçus dans cette mesure, surtout dans les câbles à faible ou moyen isolement.

Ces essais ont donc raison d'etre pour l'acheteur surtout quand ll désire s'assurer que les matières ísolantes employées sont bien d'une certaine nature, et d'une certaine qualité demandées par lui. Ce cas se présente avec les câbles au caoutchouc; un très haut isolement peut être considéré, chez ce genre de cable, comme l'indice de l'emploi de gommes où entre une proportion convenable de caoutchouc de bonne provenance a faible teneur en matières résineuses ; ces gommes de bonne qualité, et de prix élevé, donnent de bien plus grandes garanties de bonne conservit tion que d'autres mélanges de produits inférieurs, lesquels pour raient cependant pernettre, dans certains cas, de subir d'une manière en apparence satisfaisante les essais de rigidité dílec trique qui auraient étél prescrits.

Pour les câbles au papier, la situation est très différente, car les hautes valeurs de l'isolement kilométrique ne sont pas du tout 
lindice d'une meilleure fabrication ni de lemploi d'une matière londice deure qualité. Linverse serait plutôt vral, car les hautes de melleure l'isolement (telles que celles autrefors prescrites) sont valeurs d'indıce de l'emplos d'une matıère d'imprégnation chargée plutot limus résineux, peu plastique, plus facilement cassante, ef de piont à la fors moins de rigidıté diélectrıque, moms de régularité dimpnégnation, et moms de résistance aux efforts mécaniques lors du transport et de la pose.

Ce fait est maintenant universellement comnu, et la généralité dabricants a renoncé aux mélanges qui leur permettaient dobtenir des isolements kilométriques de plusieurs milliers de uégohms, et se contentent dobtenir quelques centaines, et même quelques dizanes de mégohms. Dans ces conditions (et une valeur quelconque de l'isolement au-dessus de quelques mégohms ne prísentant en elle-même pour l'exploitant aucun intérêt, mème dans le cas de très hautes tensions), ll semble préférable de s'abstenir de toute prescription à ce sujet. L'mposition soit d'une limite inférieure, sort d'une limite supérieure, ne peut que gèner mutullement le fabricant dans le chorx de ses matières isolantes et contrarer la marche du progrès.

On concevrat mieux la prescription de maintenir la valeur de pisolement entre deux limites relatives en vue d'assurer l'uniforinte de la fabrication. Mais, dans ce cas, ll faudrat avoir soin de faire tous les essais dans des conditions de température auss voisines que possible. Etant données, d'ailleurs, les nombreuses circonstances qui peuvent influer sur la valeur de lisolement sans que la qualité des câbles ne puisse en aucune façon, être incrinméc, il nous parait difficle de donner à des prescriptrons de ce denre un caractère absolu, à moins d'adopter deux limites très élongnées, c'est-à-dire d'enlever toute valeur réelle à cette prescrution.

En conséquence, pour les cảbles autres que ceux en caoutchouc, nous proposerons d'abolir toute prespription relative à la valeur de lisolement, et de n'effectuer les mesures correspondantes qu'd thre de renseignement documentaires sur la rógularté de la labrıcatıon. Les essais d'isolement dorvent, de préférence, être effectués aussitôt après l'essai sous haute tension, de manière à permettre de constater que ce dernier n`a donné lieu à aucune alteration ni à aucun échauffement anormal de la matıère isolante.

Essuis de rgidlé diélectuque. - Les essais sous haute tension a la rupture dı diélectrique sont, de beaucoup, ceux qui intéressent le plus vivement l'acheteur, car lls constituent le controle dhrect des aptitudes les plus essentielles des canalisations souterranes.

Sous quelles tensions dorvent etre effectués ces csais et quelle dot être leur durée d'application ? Les opinions sont très varıables sur ce point; les uns préconisent des essals sous tensions très supérieures à la tension normale de service; les autres estiment préf́rable, au contraire, de ne dépasser cette tension que très molérément. Toutefois, si l'on examine les choses de plus près, on constate que cette divergence est plus apparente que réelle. Qlle réside surtout dans la confusion qui s'établit parfois entre le coefficient de siécurité intrinsèque que doivent présenter les cables souterrains et la manière dont il convient de constater limportance de ce coefficient.

Tous les spécialistes sont maintenant d'accord pour reconnaitre que les cábles souterrains doivent présenter un coefficient de sécurité très considérable, c'est-à-dıre être isolés de telle façon que la rupture de l'isolant ne puisse se produire que sous une tension très considérablement supémeure à la tension normale de service.

La chose est d'ailleurs bien naturelle. Quand ll s'agıt de la résistance mécanique des matériaux, tels que le fer et l'acier, l'usage général est d'admettre un coefficien de sécurité d'au mons 4 à 5 , alors qu'on a cependant affaire à des matériaux parfaitement homogènes et identiques à eux-mêmes, et que les efforts maxima tels que ceux résultant de l'application de charges statiques sont aussi bien comnus que possible. Quand il s'agit de matériaux moins homogènes et réguliers dans leurs qualités physiques et d'eflort moins exactement connu, le coefficient de séćcurıté est tonjours considérablement augmenté et porté souvent à 8, 10 et meme au-dessus.

C'est là précisément le cas des canalisations souterraines dont les isolants sont constitués au moyen de matieres organiques complexes dont l'identité à un échantillon type ne peut jamais être complètement assurée dans toutes leurs parties, et qui doivent résister à des efforts maxima difficiles à pnévoir d'avance et lais- sant toujours place ì un très grand alía. En outre, le cuefficient de sécurité, tel qu'll est constaté aux usmes de fabrication, ne peut, comme nous l'avons défà falt remarquer, que s'atlaibln dans la suite, du fait des diverses manutentions dont les cables sont l'objet jusqu'au moment de leur entrée en service.

Ces considérations justifient done ladoption, pour les cibles souterrains, d'un coefficient de sécurité ćlevé, d'au moms 6 a 8 pour les cables de 6000 à 12000 volts, et encore plus ćlevé pour les cables fonctionnant a plus basses tensions. On y a d'autant plus de motıfs que, gràce aux progrès réalisés dans ces demiers temps, ces coefficients de sécurité élevés peuvent s'obtenr sans aucune exagération des prix de revient.

Pour apprécier ce qu'il convient de considérer comme le coefficient de sécurité, 11 est bon de porter son attention sur les tarts sulvants qui contribuent à mettre beaucoup d'indetermmation sur la valeur vértable de ce terme.

Tout le monde salt qu'en soumeltant d des essais sous tension des tronçons de cảbles de fables longueurs, on constate en général des résistances sensıblement plus considérables qu'en essayant des bobmes complètes de grande longueur. Ce faut pruvient de ce qu'un cáble savarie d'abord en ses points faubles or, malgné toutes les précautions prises, on ne peut éviter que, par suite de petites irrégularités dans la fabricalion, un câble ne présente quelques points plus fables que la moyenne. Plus la longueur soumise à l'essal est grande, et plus on a de chances de rencontrer les points de résistance mmma, et plus la tension produsant la rupture sabaisse. Le coefficient de sécurité doit s'entendre par rapport au point les plus fables, pusque ce sont ceux-là qui fixent la véritable valeur de la résistance d'm càble.

Actuellement, avec les jrogrès et les soms muntıeux apportes dans la fabrication, on est arrvé à rédure considír rablement les écarts entre les tensions de claquage des divers froncons d'w cable ; dans une bonne fabrication, on peut compter yte ces écarts ne dépasseront pas 15 à 20 pour 100 . Cette regularito permet, tout en conservant le mème coefficient de sécurité aux points les plus farbles, de l'abaisser pour la moyenne du cable. Elle est done très importante pour la réduction des prex do revient.

Un autre pomt sur lecuel ll convient dattrer lattention resido dans l'mfluence très marquéce de la durce dapphcation de la haute tension sur la rupture des isolants du genre de ceux qui entrent dans la constitution des cables.

Les phénomènes qui se produisent au voismage de la rupture dans les diélectriques soumis à des tensions très élevies sont encore peu connus et n'ont guère été étudiés expórmentalement que pour quelques diélectrıques homogènes.

Pour l'air, ll a été reconnu que, pour une certane valeur de la tension électrıque à la surface d'un conducteur, dépendant de la pression, de la température et des diverses condilons physiques de l'atmosphère ambiante, l'arr cessaut de se conporter comme un sisolant au vossmage du conducteur. Celui-cı apparaîl dans l'obscurité comme entouré d'une game lummeuse, el ce phénomène est accompagné d'un dégagenent de chalcur et d'un accroissement très sensible de la déperdition d'énergie par la surface du conducteur, surtout dans le cas des courints allematifs de fréquences élevées. L'air, dans cette régıon, parait être devenu lui-meme conducteur, et le dégagement de chaleur peut alor's s'expliquer par la circulation, a traver's cette gane conductrice, des courants de capacilé. Le phénomène s'accentue au f'ur et ì mesure que le voltage augmente, mais l'are ne s'amorce vérıtablement entre conducteurs que quand ce voltage est devenu tol que la tension électrique a dépassé la valeur crituque pour toutes les parties de l'atmosphère qui les stopare

Dess phénomènes de même genre dorvent, probablement, se produre dans tous les diélectriques; mars ils deviennent naturellement bien plus complexes quand ceux-ci, au hou d'etre parfaitement homogènes comme lair sont constutués au moren du plusieurs matières organiques. Dans ces conditions, la répartition du potentiel à l'intérieur de l'isolant peut ne plus suive de lois simples et les tensions critiques penvent ne pas etre partout les mêmes ; elles peuvent être dépassées par places en donnant lien $\grave{a}$ des ruptures partielles de l'isolant, cette rupture ne devenant totale avec production d'un véritable court circult que grand la tension électrıque a dépassé la valeur critique pour toutes les parties de l'isolant entre les conducteurs de polarités opposées.

D'autre part, il ne faut pas oublier que la rigidité diélectrique des isolants varie en sens inverse de lour température. Flle est diminué par les échauffements sensibles qui se produisout aux très hautes tensions du fait des pertes par conductibilité (laquelle 
croit elle-mene tris vile atvec la tempcirature) et du fat des pertes par hystérésis drélectrqque trés appréciables avec la plupart des isolants usuels.

Ces deux calćgories de pertes crolssent, à température égale, cumme le carré de la tension. Elles augmentent donc très rapidement.

Au dela d un cerlam voltage, à ces causes dechaulfements vemnent s'ajoutur les phénomènes calorıfiques sprciaux qur se produsent quand la tension électrique dópasse eli ccrtames partıes la valeur currespondant à la rigidité dićlectrique en ces points. L'élćvation de température r'ésultant de ces diverses causes est d'autant plus sensible que la dureé d'application du haut voltage est plus grande, et elle a pour elfet de rendre plus facle la rupture du surplus de l'isolant. Enfin, il n'est pas umpossible qu'au dela d'une certane valeur les hautes tensions mème nlternatres naient une mlluence sur lorganisation chmique et physique de certanes calígories dirzolants, de mome que les elforts mécantques domnent lieu, dans les matérlaux, à des díformatıons permanentes aussilot que la imite délasticité est dépassće, el longlemps arant que soit alteinte la limale de lupture.

En fait, el quel qu'en soil le motıf exact, on constate qu'un cable séchaufle géncralement beaucoup lorsquil est soums à une tension volsme de sa telssion de rupture, et que cette rupture peul ne pas se produre si la tension nest mamlenue que peu de temps, alors qu'elle se produirat infalliblement si cette dermère élant appliquée phis longlemps, le cable semblant perdre progressivement de sa capacitín de résistance.

Ces contestalions expliquent comment des surtensions très importantes, mais de très courle durée, peuvent ne pas produre la. rupture immédiate d'un càble, mais aussi comment elles peuvent, en étant souvent répétées, en causer l'alfablissement graduel par place jusquà un moment où ce cáble arrive à se rompre.

Elles montrent aussi pourquoi il faut évitor de soumettre un (àble à des telısıons d'essai excessives, et elles explıquent la répugnance de beancoup de constructeurs à l'application de telles tensions, surtout quand elles dorvent ctre prolongées longtemps. Il est maintenant hors de doute que de lels essais constituent pour les isolants une fatigue frès séreuse, et qu'lls sont susceptıbles, quand lis ont clés exagérés, de les détériorer d'une manière permanente.

En conséquence, si lon pouvait arrıver à assurer une régularılé de la fabrication aussı parfarte que celle réalısée, par exemple, en métallurgie, meux vaudrait évidemment éviter ces essais sur Ios cables deslinés a entrer en service, et se contenter de les effectuer sur des échantillons prélevés ad hoc. Toutefors, dans la situetion actuelle, mous pensons que ce serat là une mesuro très dangereuse et de natnre a enlever toute garantie a l'acheleur ; il nous semble mróferable, jusqu ì nouvel ordre, de conserver les essais actuellement en usage sur l'ensemble de la fabrication, mais en ćvitant toute exagération nuisible dans les prescriptions d'essais, et en ayant som de toujours les proportionner convenablement aux facultés de résistance du matériel à essayer, c'està-dire, en définiluve, all prix qu'on a consenlı à payer.

Nous ajouterons que les tensions maxima d'essai ne devront ohre maintenues que peu de temps, et que la valeur de ces tensions devra etre diminuée au fur et à mesure que leur durée d'application sera augmentée. En aucun cas, les tensions maxima. dessai ne devront soumettre aucune partie de l'isolant it une fatıgue dangereuse : elles devront réserver encore aux points les plus fatigués un coefficient de sécurité raisonnable par rapport à celles susceptibles de produre certainement la rupture, d'après les expériences faites sur l'isolant employé. En d'autres termes, il conviendra de prescrire des épaisseurs minima d'isolant en rapport avec la sévérité des essais prescrits.

Pour plus de sécurité, l'importance de la marge ainsi réservée lors des essans sous tonsion maxima devra être vérifiée sur des ichantillons de faibles longueurs pour lesquels la tension 'd'essai sera poussce jusqu'a ce que le claquage effectif se produise. Ces échantillons ctant orfinainement prélevés aux extrémités des bobines où l'imprégnation a chance d'être mieux faite, il sera en outre pirudent de vérifier que les résultats obtenus sur des échantullons prélevés dans le corpss d'une bobine sont similaires.

Enfin, pour la détermination des tensions à adopter pour les divers essais envisagés, il est bon de prendre en considération les trois points suivants :

$1^{\circ}$ Le coefficient de sécurité conslaté aux usines de fabrication devant couvrix nom semlement les alcas d'ordre électrique, mais aussi les causes d'aflablissement dorare mécanique provenant des manutentrons auxquelles le cable sera soumis durant sa mise en place, il est logique que les essais en usme solent plus sćvères que les essans après pose.

$2^{\circ}$ Par suite de considérations de fabricatıon, les épalsseurs d'isolant ne peuvent etre clmmnuces au-dessous d'une certaine limite. Il en résulte que, pour les cables à bas vollage, ces ínensseurs sonl proportromellement plus forte que pour ceux à hant voltage. Les essars devront tentr campte de ce faul, et etre propor. tomnellement plus sévères pour les cabless a bas voltage.

$3^{\circ}$ Les surtensions momentances auxquelles les củjles son exposés ne sont pas toutes proportionnelles a la tension. Celles qui peuvent se produre à la fermeture du circuit, et dont l'mpostance ne peut dépassel au maximum le double du voltage nomal sont seules dans ce cas; au contraure, les surtensions consicutwves à l'ouverture du circult, aux productions de courts-circuls aux décharges almosphérıques (c'esl-à-dıre les plus dangereuses), ont une importance relative beaucoup mondre pour les cables a très haute tension que pour ceux it basse et moyenne tension. C'est un molif de plus pour admettre un coefficient de sécurité mondre et des essars proportronnellement moins sévères pour les cables de tensions croissantes.

En passant, nous ferons remarquer que, pour ce même motif, il convient de protéger, au moyen de limiteurs de tension, surfout los cables à basse et moyenne tension. Aux hautes tensions, ces apparcils de protection deviennent d'un fonctionnement beaucoun plus délicat, sont beaucoup plus coûleux et encombrants, et ont sn outre beaucoup moms d'utllté.

Valeurs à adnettre pour les tensions dessai - Presque tous les fabricants sont acluellement d'accord pour admettre, apres pose, un essai au double du voltage de service, maintenu pendant une demi-heure a une heure, c'est-i-dire durant un laps de tempins suffisant pour permettre à tous les défauts de se révćler line teile prescription est d'autant plus logique qu'elle correspond th peu près aux essais imposés pour le matériel électrıque alimentant la canalisation, et que les câbles doivent rationnellement présenter un coefficient de sécurite au moins égal (smon supérieur) à celu du matériel des stations qu'ils desservent.

Pour ce qui concerne les essais en usme, les avis sont plus partagés, ansi que nous l'avons déjá fait remarquer ; néamonns. la tendance générale est plutot d'admetlre des essaus beancoup plus stévères que ceux qui étaient de règle il y a quelques années. Pour les voltages usuels de 5000 à 10000 volts, beaucoup do massons consellent un essai prolongé au triple du voltage aver pointes momentances au quadruple ; en particulier, l'essar al triple du voltage appliqué pendant un quart d'heure a élé recom. mandé par le Syndicat professionnel des Industries électrugues pour les câbles au-dessus de 5000 volts, et l'essar au quadruple pour les cables au-dessous de 5000 volts ; fort peu de maisons (en général étrangères) conselllent de descendre au-dessous de 2.5 fors le voltage pour l'essai de durée, et encore plusieurs de cellesth admettent-elles que, dans certains cas où une siécurité plus grande est recherchée, il peut convenir d'aller au delà, sauf bıen entendu, à augmenter en conséquence les épaisseurs d'ısolant eł le prix du cảble.

L'expérience a montré que l'augmentation de la sévérté des essais en usine a eu des résultats très favorables pour le bon fonctionnement en service courant, et qu'elle a amené, sinon la disparition complète, du moins la très grande réduction des accidents. Il nous parait donc préférable de rester dans cette vose, sauf à tenir compte des précautions dont nous avons montré ci. dessus l'utillté.

Pour donner notre opınıon personnelle, nous résumons cl-après les conditions d'essai, et les prescruptions diverses que nous croyons devoir conseiller aux intéressés pour les câbles destinés à fonctionner sous tensions supérieures à 2000 volts.

Cables au papier : essais en usine. - Les bobmes entières seront soumises pendant au moins un quart d'heure à une tension d'essai égale à deux fois la tension de service, augmenlée d'une quantité fixe de 10000 volls, c'est-à-dire déterminće par la formule suivante $\mathrm{V}=10000+2 \mathrm{E}$, dans laquelle $\mathrm{V}$ est la tension efficace d'essai, et $\mathrm{E}$ la tension efficace de service.

Dans le courant de cet essai, la tension devra ctre progressivement augmentée de 33 pour 100 au-dessus de cetle valeur, maintenue pendant environ 1 minute à cette valeur maxima. puis ramenée jusqu'à la fin de l'essai à sa première valeur. Il est préférable que l'application momentanće de la tension maxima n'ait pas lieu à la fin, mais piutôt au commencement, ou vers le milieu de l'essai, de telle sorte que la tension normale d'essai 


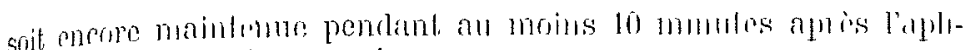
caliun de la tension mavina.

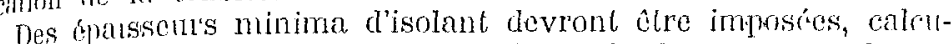
tos de telle façon que l'isolant ne travalle dans aucune de sés parties a plus de 75olo volts par millimitro lors de l'essai de duréc,

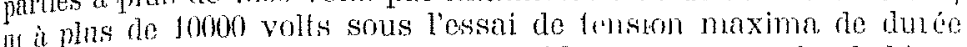

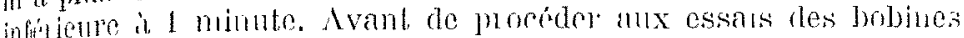

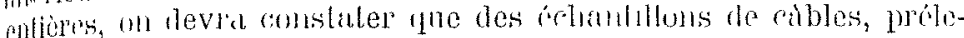

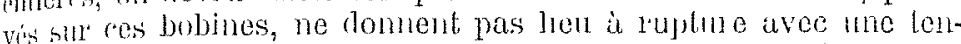
sion supereure d'an moins 50 pom 100 a la fension maximn presspite, celte tension clant appliquée graduellemenl eu partant fle lo telusion maxima prescrite ef l'augmentant, de 10 pour 100 chanur Aern-mimule jusqu'ì ce que la rupture se produsse.

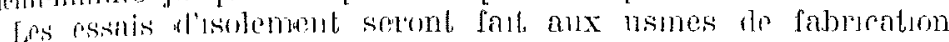
gissilit apres les essass sous lension, ef antant quo pussible dans

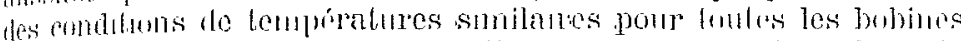

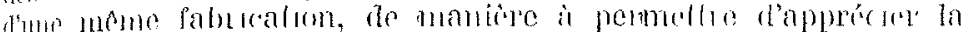

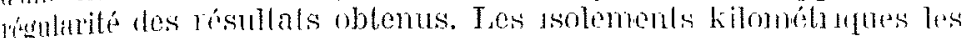

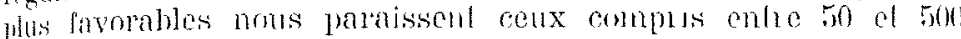

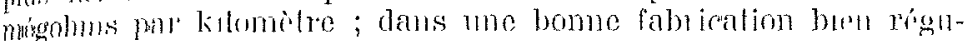

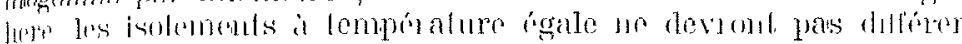
do pluts de 20 it 25 pour 100 puur les cảbles it 50 mégohms, wi de

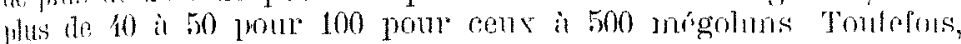

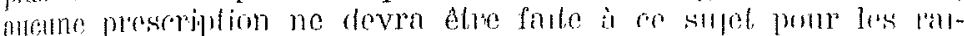
sont gute nous avoms déja expostios.

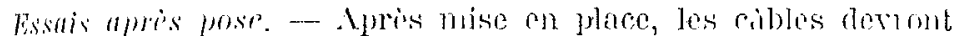

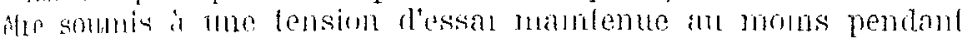
thene an duuble de la lension de setvire. Il ne sera pas man. fols. poul aulanl que les cireonslances le permetlont, de ne pro-

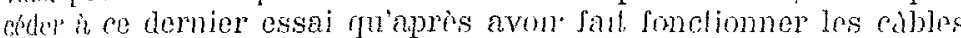
for rourt-sil coul pendant 2 ou 3 heures it un rigme de comrant

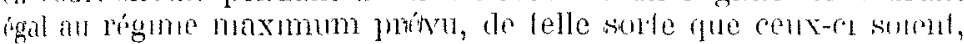

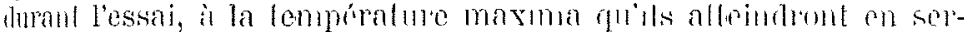
vire courant.

Les diflétenles fonsions d'esstis anx usines, el anries nose, indi-

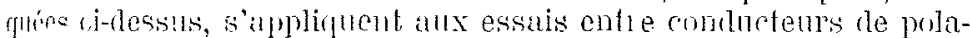

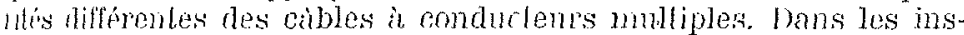

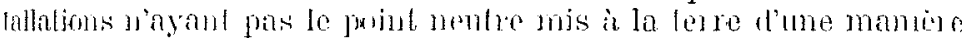

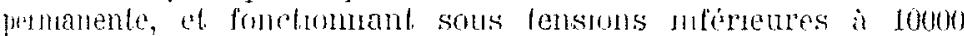
m 12000 volts, les momes essars aux memes lensions tevront the effectites entre conductem et enveloppe en plomb.

ponr les voltages supérieurs it 12000 volls, el mfémeuns it 2000 volls, ces lonsions pouront dre zividuiles de 10 pomr 100

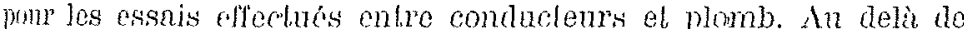

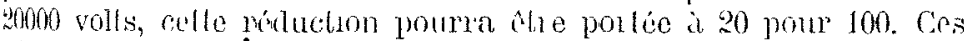
léductions sont juistifiables aux lris haules lensions, parce que la capacili du resenu mend alors me telle valeur fue le point nonle se lrumve funjours i pen pres an potenthel de la terre. et

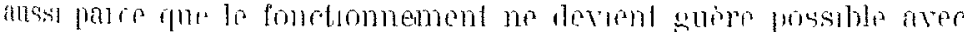
the defant lisolement sut une phase.

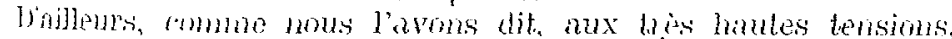

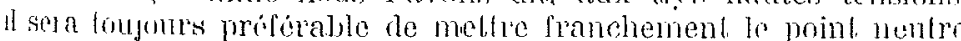

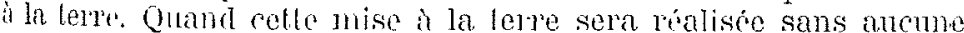
interealation re misistance, les tensions prescrites pourronl dro Irdules de 30 pour 100 pottr les essais entre conducteurs of Flomb. Quant il y amo une rósistance intercalón dans la $\mathrm{com}$ nesion is la terre, Ja réduction possible pourra varier to 20 a 30 pour 100 snuvant l'momprlaner de celle rísistance.

f'ables ramished rambric et an caoulchour. - Los cibles Yarnished cambric offrent, par leur fabrication mome, plus de ganante de rogulantí que les cables an papier, of ils sont moins sujels a se délípiorer durant la mjse en place : on oulre, ils ont

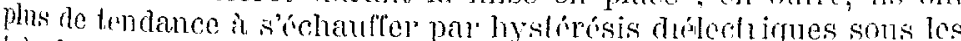
hi's hantes tensions. Pour ces deux raisons il sma preforable de

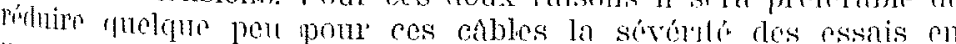
istnes. Par exemple, la formule $: V=10000+2 \mathrm{~V}$, pouma nnoir som lerme constanl réduil, et atro remplacie par la formule $\mathrm{V}=5000+2$ E. A part cette moulification, fontes les antres rondilions d'essai rléja indiquées pour les cibles au papier pourlint ctre conservées sans changement.

Pour des motifs similaires, les tensions d'essais des cobles an choutchouc pourront ctre m pen moindres que celles consenllies poun les cables an papier, surtout quand los essais seronl prosrrits apres immersion dans l'ean : ces fensions pourront atre los manps que colles indiquées pour les rables rambrir.

Enatssours minima disolaml. - Noms terminerons co chapilte

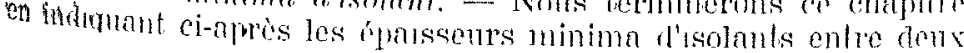

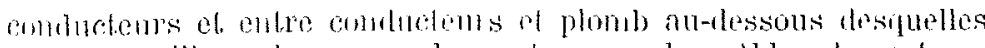

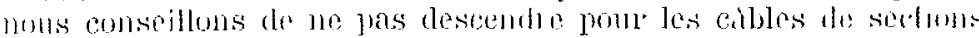
commises colve 30 at $150 \mathrm{~mm}$. par combucteurs, devant fonchmnep it tensions de 5000 volts et au-dessus, el être summis aux essais prescrits ci-clessus. Cess molications sont valables aussi ben

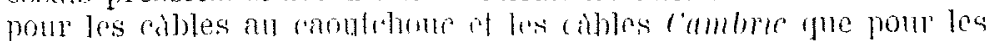
cibles nu papion.

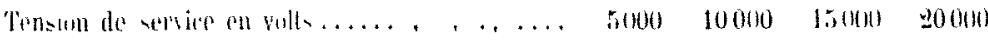

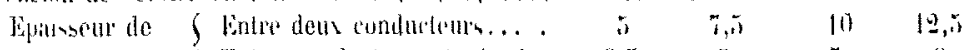

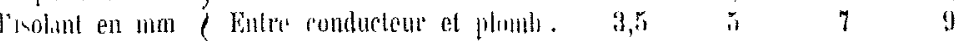

(s)iners.

\section{EXPOSITION DE MARSEILLE}

\section{(Suite)}

\section{MINISTĖRE DE L'AGRICULTURE}

La Direction de l'ilydraulique et des Améliorations agricoles avait or ganisé deux sections très intéressantes. La première de ces sections était consacrée au Sorvice d'Etude dos grandes forces hydrauliques, la seconde au service des Amoliorations agricoles.

L'exposition du service d'Ltude des grandes forces inydrauliques, organiste par $M$. de La Brosse, ingénieur

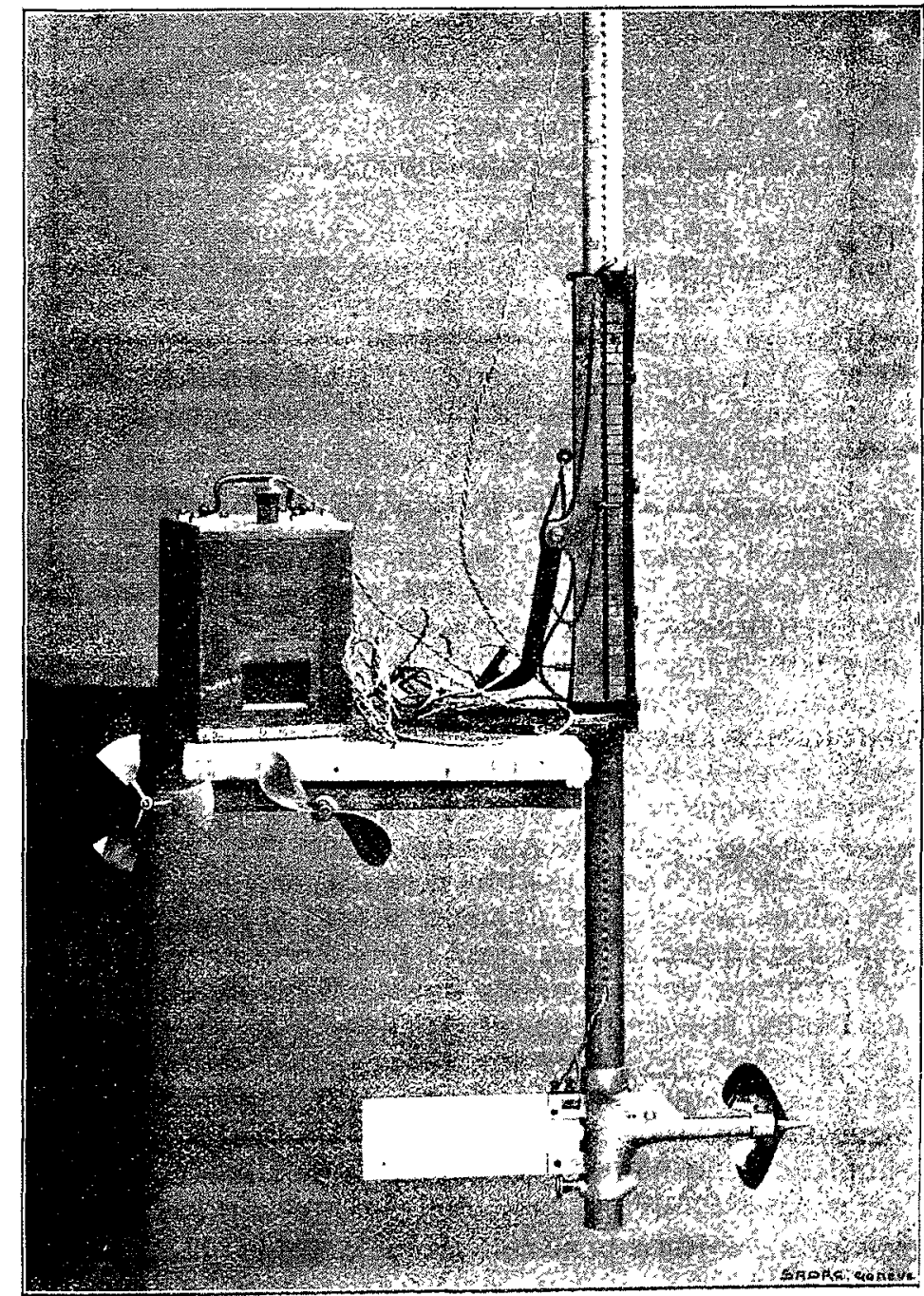

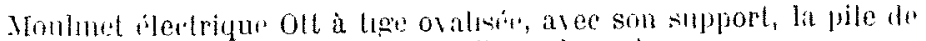
to sonnerio et sos hélues de rechango.

en chef des Pouts el Chausscies, chef de ce service d'étude pour la rugion des Alpes, était des plus remarquables, ct comportail 58 pièces, réparlies en 4 groupes.

10 Le groupe des modeles comprenait un rolief au $1 /$ hol de la station do jaugeage du pont de la Beaume, sur la Durance, a sisteron; un relief au $1 / 500$ re 19 station de 